\title{
Feasibility of Using Interactive Voice Response to Monitor Daily Drinking, Moods, and Relationship Processes on a Daily Basis in Alcoholic Couples
}

\author{
James A. Cranford, Howard Tennen, and Robert A. Zucker
}

\begin{abstract}
Background: Daily process research on alcohol involvement has used paper-and-pencil and electronic data collection methods, but no studies have yet tested the feasibility of using Interactive Voice Response (IVR) technology to monitor drinking, affective, and social interactional processes among alcoholic (ALC) couples. This study tested the feasibility of using IVR with $n=54$ ALC couples.

Methods: Participants were $n=54$ couples (probands who met criteria for a past 1-year alcohol use disorder and their partners) recruited from a substance abuse treatment center and the local community. Probands and their partners reported on their daily drinking, marital interactions, and moods once a day for 14 consecutive days using an IVR system. Probands and partners were on average 43.4 and 43.0 years old, respectively.

Results: Participants completed a total of 1,418 out of a possible 1,512 diary days for an overall compliance rate of $93.8 \%$. ALC probands completed an average of 13.3 (1.0) diary reports, and partners completed an average of 13.2 (1.0) diary reports. On average, daily IVR calls lasted 7.8 (3.0) minutes for ALC probands and 7.6 (3.0) minutes for partners. Compliance was significantly lower on weekend days (Fridays and Saturdays) compared to other weekdays for probands and spouses. Although today's intoxication predicted tomorrow's noncompliance for probands but not spouses, the strongest predictor of proband's compliance was their spouse's compliance. Daily anxiety and marital conflict were associated with daily IVR nonresponse, which triggered automated reminder calls.

Conclusions: Findings supported that IVR is a useful method for collecting daily drinking, mood, and relationship process data from alcoholic couples. Probands' compliance is strongly associated with their partners' compliance, and automated IVR calls may facilitate compliance on high anxiety, high conflict days.
\end{abstract}

Key Words: Daily Process Design, Interactive Voice Response, Daily Compliance, Alcoholic Couples.

$\mathrm{P}$ ROBLEM ALCOHOL USE and alcoholism are conceptualized as individual-level phenomena, yet numerous studies have documented reciprocal associations of alcohol involvement with variations in the marital relationship (Leonard and Eiden, 2007; Leonard and Rothbard, 1999; Marshal, 2003). For example, husbands' and wives'

From the Addiction Research Center, Department of Psychiatry $(J A C)$, University of Michigan, Ann Arbor, Michigan; Department of Community Medicine and Health Care (HT), University of Connecticut Health Center, Farmington, Connecticut; and Addiction Research Center, Department of Psychiatry (RAZ), University of Michigan, Ann Arbor, Michigan.

${ }^{1}$ Additional details on the recruitment procedures are available from the first author.

Received for publication May 4, 2009; accepted October 13, 2009.

Reprint requests: Addiction Research Center, Department of Psychiatry, University of Michigan, Room 2418, Rachel Upjohn Building, 4250 Plymouth Road, Ann Arbor, MI 48109-2700; Fax: 734-998-7992; E-mail: jcranfor@med.umich.edu

Copyright (c) 2009 by the Research Society on Alcoholism.

DOI: 10.1111/j.1530-0277.2009.01115.x

Alcohol Clin Exp Res, Vol 34, No 3, 2010: pp 499-508 drinking predicted lower marital quality and increased marital instability over 1 year (Leonard and Roberts, 1998; see Marshal, 2003; for a review), and alcohol misuse shows strong and consistent associations with intimate partner violence (Fals-Stewart et al., 2005; see Leonard and Eiden, 2007; for a review). Amato and Rogers (1997) showed that problems due to drinking or drug use predicted marital dissolution (divorce or permanent separation) 12 years later, and findings from the National Comorbidity Survey also indicated that alcohol abuse and dependence predicted divorce (Kessler et al., 1998; also see Amato and Previti, 2003; Prescott and Kendler, 2001). The reciprocal nature of the relationship between alcohol involvement and marriage was highlighted by evidence for positive effects of spousal involvement in the context of alcoholism treatment (McCrady et al., 1986; O'Farrell and Fals-Stewart, 2003).

A long line of research has used real-time behavioral observation methods to identify the marital interaction processes that may link alcohol involvement and marital outcomes (Floyd et al., 2006; Jacob and Leonard, 1988; Murphy and O'Farrell, 1996). More recently, studies have used daily 
process methods to examine the dynamics of daily alcohol involvement and relationship processes (e.g., Fals-Stewart et al., 2005). Daily process designs typically involve asking participants to report on their thoughts, feelings, and behaviors on a daily basis (Bolger et al., 2003; Tennen et al., 2000, 2003). Daily process designs have several notable strengths. In brief, daily process methods: (i) allow for the study of behavioral processes in their natural context; (ii) reduce retrospection error and bias by measuring behavior and experience close to their real time occurrence; (iii) provide important descriptive data on the temporal course of everyday experience that can be of considerable theoretical and practical significance; (iv) establish temporal precedence and allow for reliable assessment of change over time; and (v) enable researchers to address the withinperson associations between variables of interest, and determine if these within-person relations differ as a function of between-persons individual differences (Bolger et al., 2003; Tennen et al., 2000, 2003).

Daily process methods have proven useful for the study of alcohol involvement and its correlates (e.g., Helzer et al., 2002, 2008; for reviews, see Armeli et al., 2005; Leigh, 2000; Tennen et al., 2003). For example, Fals-Stewart and colleagues (2005) used daily process methods to examine the associations between daily alcohol use, antisocial personality disorder (ASPD), and male-to-female intimate partner violence. Until the mid-1990s, researchers relied on paper and pencil diaries to study daily alcohol involvement (e.g., Rohsenow, 1982; Uchalik, 1979). For example, Poikolainen and Karkkainen (1983) recruited a sample of 49 moderate-to-heavy drinking males and asked them to record their alcohol consumption every day for 12 weeks using pocket-sized diaries. However, the use of paper-and-pencil diaries has been criticized because of difficulties in assessing compliance with daily process protocols (Stone et al., 2002; cf. Green et al., 2006; Tennen et al., 2006). As a result, investigators have become increasingly concerned over the issue of verification of daily reports (see Tennen and Affleck, 2002), and some researchers have advocated for the use of electronic diaries that allow for verification of the date and time of diary reports.

Hand-held computers, wireless sensors, the internet, and the telephone have all been used as electronic data collection methods in daily process studies (Bolger et al., 2003; Shiffman et al., 2008). To our knowledge, the first series of studies that used interactive voice response (IVR) to study daily drinking behavior was conducted by Perrine and colleagues (Mundt et al., 1995; Perrine et al., 1995; Searles et al., 1995). In one study of 51 men, Mundt et al. used an IVR system to collect data on daily alcohol involvement for 112 days. Participants were asked to call a toll-free 800 number with a touch-tone telephone every day to complete various measures of alcohol involvement, mood, stress, and physical health over the preceding 24 hours.

Over the last several years, the use of IVR in daily process studies of alcohol involvement has increased. Kranzler and colleagues (2004) used daily process methods to study daily drinking behavior in a sample of heavy-drinking adults in a trial of daily versus targeted naltrexone. Participants called in to an automated IVR system every day for 84 days and reported on their daily drinking behaviors, daily moods, and medication adherence. However, to our knowledge, no studies have yet used IVR to examine daily processes among alcoholic couples (defined as couples where one or both partners has a past 1-year alcohol use disorder). High adherence is particularly important in research on dyadic processes because the effects of marital happiness on alcohol involvement vary as a function of time (McCrady et al., 2004). If data is missing for both members of the couple on the same days, and if these days are not random, we will be less likely to capture these time-varying effects. Similarly, if one member of the couple does not complete a daily report on a given day, couple-level data are unavailable on that day. Accordingly, the purpose of the present study was to test the feasibility of collecting daily process data from alcoholic couples using an IVR system.

\section{MATERIALS AND METHODS}

\section{Participants: Treatment Sample}

As part of a larger study, alcoholic probands and their spouses were recruited from a local substance abuse treatment center ( $n=20$ couples) and from the local community ( $n=34$ couples). For the treatment center sample, we screened 559 medical charts of current and incoming patients. A total of $n=73$ patients $(13.1 \%)$ of the sampling pool) met the following criteria and were thus eligible to participate in the study: (i) DSM-IV clinical diagnosis of past 1-year alcohol abuse or dependence, (ii) currently married, (iii) 18 years of age and older, and (iv) not a threat to self or others. Among those who did not meet eligibility criteria, by far the most common reason was "not currently married" (62\% of the sampling pool; cf. Zucker et al., 2000). Other common reasons for ineligibility included (i) no past 1-year alcohol use disorder (AUD) diagnosis $(9 \%$ of the sampling pool), and (ii) did not enter treatment after the intake interview or left treatment prior to recruitment (11\% of the sampling pool).

After receiving permission from the person's primary therapist, probands who met eligibility criteria were approached at the treatment center, either before or after an individual or group therapy session, and project staff explained the nature of the study and distributed an informational study brochure to the patient. For those patients who met inclusion criteria and expressed interest in participating, an initial phone call was scheduled to further discuss the study with the patient and his/her spouse. Potential participants and their spouses were contacted by phone as soon as possible after the patient had been approached (usually within 1 to 2 days). During this initial phone call, we explained the study to potential participants and their spouses and addressed any questions about study participation. Couples who agreed to participate were then scheduled for the in-person baseline $\mathrm{T} 1$ interview. Of the $n=73$ eligible recruits, $n=20$ $(27.4 \%)$ agreed to participate. Among those who did not participate, $38 \%$ took the study brochure but did not call back; $32 \%$ refused to participate (the most common reason was "too busy"); and 19\% indicated that their spouse was unable or unwilling to participate.

\section{Participants: Community Sample}

Given the challenges of recruiting married alcoholic couples from treatment, we expanded recruitment to include alcoholic couples from the surrounding community. We used the same broad inclusion criteria described earlier, with the following exception: because resources prohibited the establishment of DSM-IV clinical diagnosis 
of past 1-year alcohol abuse or dependence, we use the Rapid Alcohol Problem Screen 4 (RAPS4; Cherpitel, 2002) to screen for past 1year AUD. The RAPS4 is a 4-item screening instrument for alcohol use disorders that incorporates 2 items from the Alcohol Use Disorders Identification Test (AUDIT; Saunders et al., 1993) and 2 items from the TWEAK (an acronym for Tolerance, Worried, Eye-opener, Amnesia, and K/Cut down; Russell et al., 1994). Research showed that a positive response to 1 of the 4 items had good sensitivity and specificity for identifying those with AUDs (Cherpitel, 2000). We retained the criteria of (i) currently married, (ii) 18 years of age and older, and (iii) not a threat to self or others. Upon contacting our offices, potentially eligible persons were immediately asked if they were (i) married or living with someone for at least 6 months and (ii) over the age of 18 . Those who met both criteria were then told about the nature of the study and the requirement that the spouse also participate. We then administered the RAPS4 by telephone.

A total of 307 persons responded to our recruitment efforts. Most of the community participants $(88.2 \%)$ were recruited via newspaper and web-based advertisements. We were unable to administer the RAPS4 to $68 \%$ of potential participants: $56 \%$ left an initial phone or email message but did not respond to further contact attempts, 33\% indicated that they were "not alcoholic" after hearing a description of the study, and the remaining number refused to participate for various reasons (e.g., partner uninterested). Of the $n=98$ who screened as eligible and completed the RAPS4, over half $(67 \%)$ screened positive for a past 1-year AUD, and of those who screened positive, $34(52 \%)$ completed the T1 interview. Reasons for nonparticipation among those who screened positive and initially agreed to participate included: unable to re-contact $(n=14)$, partner unwilling to participate $(n=5)$, refused/no reason given $(n=4)$, and no transportation $(n=3)$.

\section{Final Sample}

The final sample consisted of a total of $n=54$ couples $(20$ clinical couples and 34 community couples; 37 couples with an alcoholic male proband/female partner and 17 couples with an alcoholic female proband/male partner). Alcoholic probands and their partners were on average 43.4 (13.2) and 43.0 (14.0) years old, respectively. The majority of participants $(75.9 \%$ probands, $74.1 \%$ partners) were white. For probands and partners, the modal level of education was "some college." With respect to annual income, the median level category was $\$ 30,000$ to $\$ 39,999$ for probands and $\$ 20,000$ to $\$ 29,999$ for partners.

\section{Procedures}

At Time 1 (baseline), couples completed a series of self-report measures and an IVR training session at our research center. One week later at Time 2 (T2), couples began the daily process phase of the study. Alcoholic probands and their spouses separately called in to an automated IVR system every night between 5:00 PM and 9:00 PM for 14 consecutive nights and answered questions about (i) the previous night's drinking behavior and marital interactions, and (ii) today's drinking behavior, marital interactions, and moods. Participants reported on their drinking behaviors and marital interactions for 2 time frames: (i) last night after you completed the telephone interview, and (ii) since you woke up today.

Previous daily process studies of individual alcohol involvement have collected reports for as long as 2 years (Helzer et al., 2002), and daily process studies of alcoholic couples have collected data for as long as 15 months with good compliance (Fals-Stewart et al., 2005). We selected a 14-day period because, to our knowledge, this is the first study to use IVR to collect daily process data from alcoholic couples. The 14-day period allowed for the collection of enough data to discern daily and weekly patterns in the variables of interest without imposing undue burden on participating couples.

\section{Measures}

We were interested in assessing the within-person and betweenpersons predictors of compliance. Accordingly, we obtained within (i.e., daily) and between-persons (i.e., past 1 month) measures of moods, marital interactions, and alcohol involvement. All past 1month measures were completed during the baseline interview, so that for each participant, these reports represented their moods and behaviors in the month prior to the baseline interview.

Past Month and Daily Moods. We used an adapted 18-item version of the Profile of Mood States (POMS; McNair et al., 1992) to assess 5 past 1-month mood variables: depressed mood (4 items), anxious mood ( 3 items), anger ( 3 items), fatigue ( 3 items), and positive affect (5 items). Participants used a 5-point scale $(0=$ not at all to $4=$ extremely) to indicate the extent to which they experienced these moods during the previous month. Cronbach's alphas for probands and spouses, respectively, were 0.93 and 0.85 for depressed mood; 0.86 and 0.76 for anxious mood; 0.87 and 0.79 for anger; 0.89 and 0.89 for fatigue; and 0.93 and 0.88 for positive affect. Scores for each mood scale were obtained by averaging the ratings of the relevant items.

The same 18 items were used to assess daily moods. Participants use the same 5 -point scale $(0=$ not at all to $4=$ extremely $)$ to indicate the extent to which they experienced each mood since you woke up today. Daily scores for each mood were obtained by averaging the ratings of the relevant items. Methods outlined in Cranford and colleagues (2006) were used to calculate between- and within-subjects reliabilities for each scale. The between-subject reliability estimates are interpreted as the between-subjects reliabilities of the average of the items for 1 fixed day $\left(\mathrm{R}_{1 \mathrm{~F}}\right.$ in Cranford et al.). The within-subjects reliabilities are interpreted as the reliability of each scale for detecting systematic changes in moods within subjects over all diary days $\left(R_{C}\right.$ in Cranford et al.). For daily depressed mood, estimates of $\mathrm{R}_{1 \mathrm{~F}}$ for probands and spouses, respectively, were 0.80 and 0.86 ; and estimates of $R_{C}$ were 0.79 and 0.84 . For daily anxious mood, estimates of $R_{1 F}$ for probands and spouses, respectively, were 0.76 and 0.79 ; and estimates of $R_{C}$ were 0.71 and 0.74 . For daily anger, estimates of $R_{1 F}$ for probands and spouses, respectively, were 0.67 and 0.79 ; and estimates of $R_{C}$ were 0.78 and 0.83 . For daily fatigue, estimates of $R_{1 F}$ for probands and spouses, respectively, were 0.87 and 0.85 ; and estimates of $R_{C}$ were 0.86 and 0.81 . For daily positive affect, estimates of $R_{1 F}$ for probands and spouses were both 0.86 ; and estimates of $\mathrm{R}_{\mathrm{C}}$ were 0.81 and 0.80 . There was no discernible pattern of systematic change in the reliability coefficients from week 1 to week 2 for either probands or spouses.

Past Month and Daily Negative Marital Interactions. We used the 7-item Social Undermining Scale (SUND), a measure drawn from the work of Vinokur and colleagues (1996), and 3 items from Schulz and colleagues' (2004) Withdrawn Marital Behavior Scale to assess past-month negative marital interactions. Participants used a 5-point scale $(0=$ not at all to $4=$ about every day $)$ to indicate how frequently their spouse engaged in various negative behaviors (e.g., criticism) during the past 1 month. Cronbach's alphas for probands and spouses, respectively, were 0.93 and 0.90 . Due to concerns over participant burden, we used only 5 of these 10 items to assess daily negative marital interactions. Participants responded to each item for 2 time frames: (i) last night after you completed the telephone interview, and (ii) since you woke up today. Response options for the daily process items were 0 (no) and 1 (yes). An index of the total number of negative marital interactions for each day was computed by summing the items.

Past Month and Daily Positive Marital Interactions. Nine items taken from Manne and colleagues (2004) and de Koning and Weiss (2002) were used to assess past 1-month positive marital 
interactions (e.g., spouse acceptance). Items asked about the spouse's behavior during the past 1 month, using the same response options as those for the negative marital interaction items, and scores were calculated as the mean of the item scores. Cronbach's alphas for probands and spouses, respectively, were 0.89 and 0.87 . We used 5 of these 9 items to assess daily positive marital interactions, and the format and response options were the same as those for the daily negative marital interaction items. An index of the total number of positive marital interactions for each day was computed by summing the items.

Past 1 Month and Daily Alcohol Involvement. At T1, we used 3 items to assess past 1-month alcohol consumption (NIAAA, 2003). We obtained measures of (i) number of days consumed 1 or more alcoholic beverages (frequency), (ii) usual number of drinks per drinking day (quantity), and (iii) frequency of binge drinking, defined as consuming 5 drinks for men (4 for women) within a 2-hour period (NIAAA, 2004). In addition, the Short Inventory of Problems (SIP; Miller et al., 1995) was used to measure alcohol-related problems in the past 3 months. Participants used a 5 -point scale $(0=$ never to $3=$ daily or almost daily) to indicate how frequently they had experienced each alcohol-related problem during the past 3 months. Cronbach's alphas for probands and spouses, respectively, were 0.95 and 0.94 .

Items asking about daily alcohol involvement were adapted from Helzer and colleagues (2002) and Kranzler and colleagues (2004) using the standard definitions of alcoholic beverages (National Institute on Alcohol Abuse and Alcoholism, 2003). Also, following Kranzler and colleagues, for each IVR report participants reported on their alcohol involvement for 2 time periods: (i) since waking up today, and (ii) after completing last night's IVR report. For each time period, participants were asked to indicate how many 12 ounce cans or bottles of beer, drinks of wine, and standard drinks of hard liquor they had consumed. Variables for number of drinks since waking up today and number of drinks after completing the IVR tonight were calculated by summing responses across the 3 beverage types. We also calculated a variable for total number of drinks today by summing the values for "today's drinks" and "tonight's drinks." In order to assess daily binge drinking for the 2 time periods, participants were asked if they had consumed $[5$ or more drinks for males $/ 4$ or more drinks for females] within a 2-hour period (since waking up today and after completing last night's IVR). Response options for the binge drinking items were 0 (no) and 1 (yes).

In addition to these daily drinking measures, we also included 6 items about daily alcohol involvement from Searles and colleagues (1995) (also see Helzer et al., 2002). For both time periods (i.e., since waking up today, and after completing last night's IVR report), participants were asked to indicate their (i) urge to drink $(0=$ no urge to drink and $10=$ the strongest urge ever to drink $)$, highest level of intoxication $(0=$ perfectly sober and $10=$ as drunk as you've ever been), hangover $(0=$ no hangover and $10=$ worst hangover you have ever had), the severity of any drinking-related problems $(0=$ no problems and $10=$ extremely serious problems $)$, and the location where they drank. Finally, the last IVR question asked participants to indicate how intoxicated do you feel right now? $(0=$ perfectly sober and $10=$ as drunk as you've ever been $)$. As these 6 variables were positively skewed, we created binary versions of each variable. All participants responded to the same number of questions regardless of whether or not they reported drinking on that day.

\section{IVR Training Session}

Following completion of the marital interaction task, the project psychologist introduced Phase 2 of the pilot study, which involved completion of the daily process measures every day for 14 consecutive days using the automated IVR system. The IVR protocol for the current study was modeled after procedures described in Kran- zler and colleagues (2004), which was the first application of IVR in an alcohol treatment study. Couples completed an extensive IVR training session immediately following the marital interaction task. Following the IVR training session, couples were compensated $\$ 50.00$ for completing the marital interaction task and the IVR training session, and were provided with a study brochure with contact numbers for study staff in the event of questions or concerns. The day before the couple was scheduled to begin calling in to the IVR system, the project research assistant placed a reminder call to each couple and asked if there were any questions or concerns. Compliance with the IVR protocol was monitored daily by project staff and participants were called if they missed 2 consecutive IVR days, as recommended by Abu-Hasaballah and colleagues (2007). Dates and times of all calls into the IVR system were recorded electronically, allowing for assessment of compliance with diary protocols (Stone and Shiffman, 2002). Participants called a dedicated tollfree telephone number during the designated time window (5:00 PM to 9:00 PM) and responded to survey questions using the telephone keypad. Participants were asked to call separately when they had 15 minutes of privacy and were instructed not to discuss their responses with each other. Following completion of the 14-day IVR protocol, couples were compensated $\$ 100$ ( $\$ 50.00$ per partner, approximately $\$ 3.60$ per call). Incentives were prorated such that participants were compensated only for completed calls. A description of IVR and a summary of best practices are given in AbuHasaballah and colleagues (2007). ${ }^{1}$

\section{Comparisons Between Clinical and Community Samples}

Comparisons between the probands and their partners from the clinical and community samples showed that clinical probands $(\mathrm{M}=5.7, \mathrm{SD}=2.1)$ had a significantly higher income level than community probands $(\mathrm{M}=2.9, \mathrm{SD}=2.0), t(48)=4.7, p<0.01$ and significantly lower levels of past month positive affect $(\mathrm{M}=1.6$, $\mathrm{SD}=1.0)$ than community probands $(\mathrm{M}=2.2, \mathrm{SD}=0.8), t(52)$ $=-2.3, p<0.05$. The 2 groups did not differ on any of the other past month mood variables, all $p>0.10$. There were no group differences in frequency of past month negative or positive marital interactions. In addition, there were no significant group differences in number of drinking days, number of drinks per drinking day, or number of binge drinking days in the past year. However, community probands reported a significantly higher number of drinking days in the past 1 month $(\mathrm{M}=15.0, \mathrm{SD}=9.7)$ than clinical probands $(\mathrm{M}=2.5, \mathrm{SD}=5.4), t(52)=-5.3, p<0.01$. Community probands also reported more drinks per drinking day in the past 1 month $(\mathrm{M}=4.2, \mathrm{SD}=4.5)$ than clinical probands $(\mathrm{M}=1.8$, $\mathrm{SD}=4.6)$, and this difference approached statistical significance, $t(52)=-1.9, p=0.07$. By contrast, clinical probands reported more frequent alcohol-related problems $(\mathrm{M}=18.6, \mathrm{SD}=11.7)$ than community probands $(\mathrm{M}=10.4, \mathrm{SD}=10.5), t(52)=2.7$, $p<0.05$. Accordingly, we statistically controlled for recruitment source in all analyses.

\section{RESULTS}

\section{Compliance With IVR Protocol}

Participants completed a total of 1,418 out of a possible $1,512(54 \times 2 \times 14)=$ daily process reports, for an overall compliance rate of $93.8 \%$. Using procedures for analyzing dyadic data outlined in Kenny and colleagues (2006), we tested for differences in dependent proportions using the McNemar test. Compliance was slightly higher among probands $(94.3 \%)$ compared to spouses $(93.3 \%)$, but this 
difference was not significant, $z=1.30$. Participants completed an average of $13.1(\mathrm{SD}=1.1)$ out of a possible 14 IVR days (range $=9$ to 14 days, median $=13$ days, mode $=14$ days $)$. About half of the sample $(n=51,47.2 \%)$ completed all 14 daily IVR reports. Compliance did not vary by recruitment source.

\section{Day-Level Correlates of Noncompliance}

For each IVR day, we computed 2 binary variables: one indicating if today's IVR call was completed, and one indicating if tomorrow's IVR call was completed (both coded $0=$ Yes, $1=$ No). We estimated generalized linear mixed models (GLMMs; Raudenbush and Bryk, 2002) of today's and tomorrow's noncompliance using the GLIMMIX procedure in SAS (SAS, 2008). All GLMMs used a Bernoulli sampling model at level-1 with a logit link. The log odds of noncompliance were then linked to day-level predictors in a level-1 structural model that took the form of a logistic regression model (Agresti, 1996). Separate GLMMs for probands and spouses were estimated.

For probands and spouses, there was no significant association between day in the study and today's noncompliance. However, results showed that the odds of today's noncompliance were significantly higher on Fridays and Saturdays compared to other weekdays for probands $(\mathrm{OR}=2.6,95 \%$ $\mathrm{CI}=1.4$ to 4.8$)$ and spouses $(\mathrm{OR}=2.0,95 \% \mathrm{CI}=1.1$ to 3.6). These results did not vary by recruitment source (clinical vs. community). Results for tomorrow's noncompliance, which are presented in Table 1, showed that (i) none of today's 5 daily moods predicted tomorrow's noncompliance for probands or spouses; and (ii) total number of today's negative and positive interactions did not predict tomorrow's noncompliance for probands or spouses. We also examined the effects of today's alcohol involvement on tomorrow's noncompliance for probands and spouses, limiting analyses to those who reported drinking on at least 1 of the 14 IVR days. Results from GLMMs showed that (i) total drinks consumed today, (ii) today's urge to drink, (iii) today's alcoholrelated problems, and (iv) today's hangover did not predict tomorrow's noncompliance for probands or spouses. However, as seen in Table 1, today's intoxication predicted higher odds of tomorrow's noncompliance for probands but not for spouses.

\section{Person-Level Correlates of Noncompliance}

In order to examine person-level predictors of noncompliance, we calculated a binary variable for each participant indicating if they had missed any of the IVR days. We used a binary version of the "number of missed IVR days" variable because it showed a severe positive skew $(94.7 \%$ of participants missed 3 or fewer days). Bivariate and multiple logistic regression analyses were used to test for between-persons correlates of noncompliance for probands and spouses. As seen in Table 1, education level, gender, age, income, and years
Table 1. Day- and Person-Level Correlates of Tomorrow's Noncompliance

\begin{tabular}{|c|c|c|}
\hline & $\begin{array}{l}\text { Proband } \\
\text { noncompliance } \\
(n=54)\end{array}$ & $\begin{array}{c}\text { Partner } \\
\text { noncompliance } \\
(n=54)\end{array}$ \\
\hline & OR $(95 \% \mathrm{Cl})$ & OR $(95 \% \mathrm{Cl})$ \\
\hline \multicolumn{3}{|l|}{ Day-level correlates } \\
\hline \multicolumn{3}{|l|}{ Today's moods } \\
\hline Depressed mood & $1.3(0.6-2.8)$ & $0.8(0.4-1.6)$ \\
\hline Anxious mood & $0.6(0.3-1.3)$ & $0.9(0.5-1.6)$ \\
\hline Anger & $1.6(0.9-2.9)$ & $1.3(0.8-2.1)$ \\
\hline Fatigue & $0.7(0.5-1.1)$ & $1.2(0.8-1.8)$ \\
\hline Positive affect & $1.0(0.6-1.6)$ & $1.3(0.8-2.1)$ \\
\hline \multicolumn{3}{|l|}{ Today's marital interactions } \\
\hline Negative interactions & $0.9(0.6-1.2)$ & $1.1(0.8-1.4)$ \\
\hline Positive interactions & $0.9(0.7-1.2)$ & $1.1(0.9-1.4)$ \\
\hline \multicolumn{3}{|l|}{ Today's alcohol involvement $\mathrm{a}^{\mathrm{a}}$} \\
\hline Total drinks & $1.1(0.9-1.3)$ & $1.1(0.8-1.5)$ \\
\hline Any binge drinking & $1.7(0.2-14.1)$ & NA \\
\hline Any urge to drink & $0.7(0.3-1.6)$ & $0.9(0.4-2.0)$ \\
\hline Any intoxication & $3.0^{*}(1.1-8.3)$ & $1.2(0.2-5.4)$ \\
\hline Any hangover & $0.9(0.2-3.4)$ & NA \\
\hline Any alcohol-related problems & $1.8(0.5-5.9)$ & NA \\
\hline \multicolumn{3}{|l|}{ Person-level correlates } \\
\hline \multicolumn{3}{|l|}{ Demographics } \\
\hline Gender $(1=$ male $)$ & $1.2(0.4-3.7)$ & $1.2(0.4-3.8)$ \\
\hline Age & $0.96(0.91-1.003)$ & $0.96(0.92-1.01)$ \\
\hline Education & $0.8(0.5-1.4)$ & $1.9(0.9-3.8)$ \\
\hline Race $(1=$ non-white $)$ & $1.2(0.3-4.3)$ & $3.0(0.7-12.7)$ \\
\hline Years married & $0.97(0.92-1.02)$ & $0.98(0.93-1.03)$ \\
\hline Marital satisfaction & $0.7(0.4-1.6)$ & $1.2(0.6-2.6)$ \\
\hline \multicolumn{3}{|l|}{ Past 1-month moods } \\
\hline Depressed mood & $0.4(0.1-1.4)$ & $1.1(0.4-3.0)$ \\
\hline Anxious mood & $0.8(0.2-2.7)$ & $1.0(0.4-2.4)$ \\
\hline Anger & $1.6(0.7-3.7)$ & $0.8(0.3-1.9)$ \\
\hline Fatigue & $1.2(0.5-2.9)$ & $1.1(0.6-2.4)$ \\
\hline Positive affect & $0.4(0.2-1.1)$ & $0.9(0.4-2.1)$ \\
\hline \multicolumn{3}{|c|}{ Past 1-month marital interactions } \\
\hline Negative interactions & $1.1(0.6-2.1)$ & $0.8(0.4-1.8)$ \\
\hline Positive interactions & $1.3(0.7-2.6)$ & $0.7(0.3-1.6)$ \\
\hline \multicolumn{3}{|l|}{ Past 1-month alcohol use $\mathrm{e}^{\mathrm{b}}$} \\
\hline Frequency of drinking & $0.9(0.8-1.01)$ & $1.0(0.9-1.04)$ \\
\hline Quantity of drinking & $1.1(0.9-1.4)$ & $0.8(0.5-1.3)$ \\
\hline Binge drinking days & $0.8(0.7-1.04)$ & $1.2(0.8-1.7)$ \\
\hline Alcohol-related problems & $1.02(0.9-1.1)$ & $1.03(0.9-1.2)$ \\
\hline \multicolumn{3}{|l|}{ Past 1 -month substance use } \\
\hline Any marijuana use ( $1=$ yes $)$ & $0.5(0.04-6.5)$ & $1.2(0.9-1.2)$ \\
\hline Any cocaine use $(1=$ yes $)$ & $1.0(0.3-3.7)$ & $0.8(0.2-3.2)$ \\
\hline
\end{tabular}

The table shows adjusted odds ratios from separate multiple logistic regression analyses using sets of day- and person-level correlates as predictors of tomorrow's noncompliance for probands and their partners. Each set of variables was tested separately (e.g., variables for today's moods were treated as a separate set). Results remained the same when all variables were tested simultaneously.

$\mathrm{OR}$, odds ratio; $\mathrm{Cl}$, confidence interval; $\mathrm{NA}$, odds ratio not computed for partners because no days of binge drinking, hangover, or alcoholrelated problems were followed by noncompliance days.

${ }^{a}$ Assessed only for participants who reported drinking during the 14-day IVR period.

${ }^{\mathrm{b}}$ Assessed only for participants who reported drinking during the past 1 month.

${ }^{*} p<0.05$.

married did not predict noncompliance for probands or spouses. Trait-level measures of moods, past month negative and positive marital interactions, and marital satisfaction were all unrelated to noncompliance. Further, there were no statistically significant relationships between noncompliance and past 1-month alcohol or other substance use. 


\section{Dyad-Level Correlates of Compliance}

Procedures described by Kenny and colleagues (2006) were used to examine the association between proband and spouse compliance across all IVR days. Dyads were distinguishable by proband status, and Cohen's kappa (Cohen, 1960) was used to test the hypothesis of nonindependence of proband and spouse daily compliance. Results showed that there was a strong association between proband and spouse daily compliance, $\kappa=0.57, p<0.01$. This effect was very similar for male $(\kappa=0.60)$ and female $(\kappa=0.53)$ probands. The probability of proband compliance on a given IVR day was about 0.95 . On days when the spouse completed the IVR, the probability of probands' compliance increased to about 0.98 . By contrast, on days when the spouse did not complete the IVR, the probability of probands' compliance decreased to about 0.45 .

\section{Time to Complete IVR Calls}

The final IVR questionnaire included 48 separate items asking about daily moods, marital interactions, and drinking behaviors. On average, IVR calls took 7.5 (2.9) minutes. IVR call time decreased as number of days in the study increased for both probands $(r=-0.44, p<0.01)$ and spouses $(r=-0.44, p<0.01)$. For example, on the first IVR day, the average call time was 11.8 (2.9) minutes. By IVR day 7, the average call time dropped to 6.9 (2.4) minutes, and by the final IVR day, the average call time dropped to 6.3 (2.5) minutes. Average length of the IVR call was moderately correlated with age for probands $(r=0.30, p<0.05)$ and spouses $(r=0.33, p<0.05)$, but was not associated with sex, income, education, number of hours worked in the previous week, or number of children in the home. Further, there were no associations between average length of the IVR call and (i) trait-level measures of moods, (ii) past month negative and positive marital interactions, (iii) marital satisfaction, (iv) frequency and quantity of past 1-month alcohol use, (v) alcohol-related problems, or (vi) past 1-month cigarette, marijuana, or cocaine use.

\section{Automated Reminder Calls}

Automated reminder calls were made by the IVR system on 148 days where either the proband or spouse had not called by 8:00 PM and resulted in completed calls on 54 days $(36.5 \%)$. Spouses completed a higher percentage of daily reports after receiving reminder calls $(n=34,4.8 \%$ of all completed calls) than probands $(n=20,2.8 \%$ of all completed calls), but this difference was not significant, $z=1.91$. Probands reported more negative marital interactions on days that required a reminder call $(\mathrm{M}=2.7)$ than on days that did not require a reminder call $(\mathrm{M}=1.4), t(628)=2.6$, $p<0.01$, Cohen's $d=0.54$. Further, probands reported higher levels of anxiety on days that required a reminder call $(\mathrm{M}=1.4)$ than on days that did not require a reminder call $(\mathrm{M}=0.9), t(711)=2.4, p<0.05$, Cohen's $d=0.52$. Among spouses, days that did versus those that did not require a reminder call were also characterized by more negative marital interactions $[\mathrm{M}=2.5$ vs. $\mathrm{M}=1.6, t(613)=$ $2.1, p<0.05$, Cohen's $d=0.37]$ and fewer positive marital interactions $[\mathrm{M}=5.9$ vs. $\mathrm{M}=7.0, t(613)=2.0, p<0.05$, Cohen's $d=-0.36$. There were no differences between days that did and did not require a reminder call on any measure of alcohol involvement.

\section{DISCUSSION}

The purpose of the present study was to test the feasibility of collecting daily drinking, mood, and marital interaction data from alcoholic couples using an IVR system. Previous work using IVR technology to study alcohol involvement has reported good compliance rates (e.g., Helzer et al., 2002; Searles et al., 1995, 2000), even among participants with alcohol use disorders (Simpson et al., 2005; Tucker et al., 2007). Several studies used daily process methods to collect data from alcoholic couples (Fals-Stewart et al., 2005) and couples with male social drinkers (Perrine et al., 1995). For example, Perrine and colleagues used IVR to collect daily process data from a sample of 30 male social drinkers for 28 days. Perrine and colleagues also asked participants' partners to report on the participant's daily alcohol consumption by calling in to a voice-mail answering service. However, to our knowledge the current study is the first to use IVR technology to collect data from both partners in alcoholic couples. Our results showed that daily compliance exceeded $90 \%$ for probands and their spouses. Previous research using daily process methods has emphasized the importance of extensive training sessions (e.g., Mundt et al., 1995), and this appears to have facilitated couples' compliance with the study protocol.

Several predictors of daily compliance were observed. Lower compliance on the first IVR day may have been due to the 1-week interval between the T1 interview and the start of the IVR protocol. Although this interval was selected in order to minimize possible carry-over effects of the T1 marital interaction task on the IVR reports, future research could formally test this hypothesis and/or select a shorter interval (see Merrilees et al., 2008). Lower compliance on Fridays and Saturdays was also observed, which may have been due to the higher levels of alcohol consumption on weekends. Also, departure from normal routines on weekends may have increased the likelihood that participants would forget to call. IVR training sessions could (i) emphasize the importance of completing IVR reports on weekend days and (ii) consider using an expanded time window to enhance compliance.

Given that several daily process studies of alcohol involvement have focused on moods and/or marital interactions (Fals-Stewart et al., 2005; Kranzler et al., 2004), it is encouraging that none of these variables predicted noncompliance, either at the between- or within-persons levels. However, we found that today's intoxication predicted tomorrow's noncompliance among probands (but not spouses). Litt and 
colleagues (1998) also found some evidence that alcohol consumption has a negative effect on daily compliance in their ecological momentary assessment (EMA) study of 27 alcohol-dependent men. Litt and colleagues found that almost half of their participants who reported drinking during the study period showed deteriorations in compliance on subsequent days. One possible solution to this problem is to closely monitor participants' reports of intoxication and initiate personal reminder calls on subsequent days. We emphasize, however, that none of the other measures of alcohol involvement predicted noncompliance.

The strongest predictor of noncompliance was the spouse's noncompliance. This finding is consistent with research on Behavioral Couples Therapy (BCT) for alcohol use disorders. For example, McCrady and colleagues (1986) found that greater spousal involvement in alcohol treatment was associated with higher treatment compliance (i.e., more treatment sessions and a higher proportion of completed conjoint homework assignments). However, given that daily marital interactions were not predictive of noncompliance, the association between proband and spouse compliance may have been driven by couples' shared activities during the evening that conflicted with the IVR time window. This finding highlights the importance of emphasizing both partners' compliance during the IVR training session.

Automated reminder calls were useful for collecting data from probands and spouses alike. Kranzler and colleagues (2004) also found evidence for the effectiveness of automated reminder calls in an IVR study of heavy drinkers, and Corkrey and Parkinson (2002) reviewed several studies showing that automated IVR calls increased appointment rates and treatment compliance (also see Abu-Hasaballah et al., 2007). Our results showed that higher levels of anxiety and marital conflict were associated with failure to call the IVR system within the designated time window, which in turn triggered the automated reminder calls. Although these findings suggest that automated reminder calls might be useful for enhancing compliance on days characterized by higher levels of anxiety and marital conflict, we were not able to directly test this hypothesis. Yet, given the relatively high levels of negative marital interactions among alcoholic couples (FalsStewart et al., 2005; Floyd et al., 2006) and the elevated rates of anxiety disorders among alcoholic probands and their relatives (Schuckit et al., 1997), these results demonstrate the value of automated reminder calls to study moods and marital dynamics in this population.

\section{Limitations and Strengths}

Results from this research should be considered in the context of several limitations and strengths. We used a small convenience sample that included probands currently in alcohol treatment and community probands not in treatment. Community probands met screening criteria for a past 1-year alcohol use disorder, but in the absence of clinical assessment we were not able to confirm participants' clinical status. Also, participation rates for both groups were relatively low, and the extent of nonresponse bias is unknown. In addition, while reports on today's and last night's drinking behaviors and marital interactions were relatively close to the real-time occurrence of daily behaviors, they still allow room for retrospection biases (see Stone et al., 1998; Tennen et al., 2000). Also, it is possible that some partners may have completed the IVR reports for their spouses. To minimize this possibility, we explicitly instructed participants to call the IVR system separately when they had 10 to 15 minutes of privacy. Also, each partner was given a unique identification number to login to the IVR system. Further, during the IVR training session, each partner was asked to log-in to the IVR system using their own identification number and complete at least 5 sample items from the daily diary questionnaire. Despite these precautions, we acknowledge that it is impossible to determine if spouses completed their partners' IVR reports.

Several strengths of the present study are noteworthy. To our knowledge, this is the first study to use IVR to collect daily process data from both partners in alcoholic couples. As noted earlier, compliance takes on added importance in research on couples because data from both partners are needed to model dyadic processes (e.g., Dunn et al., 1987). Although our sample was diverse, we were able to demonstrate that IVR can be used to collect daily drinking, mood, and marital interaction data from alcoholic couples with good compliance. Verification of the date and time of daily reports is also an important concern in daily process research (Stone and Shiffman, 2002), and by using IVR to collect daily process data we were able to confirm the dates and times of all IVR calls. Also, our design allowed us to identify betweenand within-persons predictors of daily compliance and to test for dyadic effects on compliance.

\section{Future Directions and Implications}

Findings suggest several directions for further research. Identifying methods for detecting fraudulent reporting (i.e., partners completing each other's IVR reports) is a research priority. In order to maximize the probability that probands and partners would complete only their own (and not their partner's) IVR reports, we used written and verbal instructions, unique identification numbers for each proband and partner to log-in to the IVR system, and intensive training sessions. In addition to these methods, some form of voice recording may be the optimal way to detect fraudulent reporting. Also, in the current study, probands and partners reported on their own moods and behaviors, and future research can benefit by obtaining collateral reports of probands' alcohol involvement (cf. Dunn et al., 1992; Perrine et al., 1995). In addition, the relatively high compliance rates among couples in the present study suggest that IVR might be useful for collecting daily process data from other family members, including children. Recent work demonstrated the feasibility of using IVR technology to collect data from children as young as age 9 (Stritzke et al., 2005), and using IVR 
to collect daily process data from children living in alcoholic families would be a logical next step. Finally, for some phenomena (e.g., moods), ecological momentary assessment (EMA) involving multiple assessments within days may be needed to capture the dynamic processes of alcohol involvement and its correlates with adequate temporal resolution (Shiffman et al., 2008). Collins and colleagues (2003) demonstrated the feasibility of collecting EMA data on alcohol involvement using cellular phones with an IVR system, and this represents a promising direction for research on alcohol involvement in couples.

Daily process methods have been used to study relationship processes in nonalcoholic (Rafaeli et al., 2008) and alcoholic couples (Fals-Stewart et al., 2005). To our knowledge, this is the first study to use IVR technology to collect daily process data from alcoholic couples, and findings indicated that IVR is feasible and yields good compliance with a once-daily protocol.

The potential significance of the current study is directly tied to the observation that risk and protective factors for alcohol problems are dynamic processes that operate in real time and across developmental and socio-historical time (Zucker, 1994). As noted earlier, a substantial body of research using behavioral observation methods has identified some of the real-time marital interactions that characterize alcoholic couples (e.g., Floyd et al., 2006; Jacob and Leonard, 1988). In addition, several long-term prospective longitudinal studies following participants for up to 40 years have advanced our understanding of longer-term trajectories of alcohol involvement (see Zucker, 2008). At the same time, social psychological influences on developmental course and outcome are poorly specified in current typologies of alcoholism (Zucker et al., 1995). Daily process studies can yield invaluable evidence for the linkages between real-time and developmental time processes and our results indicate that IVR technology can be used to better specify the marital context of alcohol use disorders.

We provide 2 examples of how daily process methods and IVR technology can advance research on marital interactions and alcohol involvement. First, real time characterization of marital interactions is critical to the understanding of marital processes at a micro-level. At the moment, our science is constrained by the fact that such data are usually collected in the artificial setting of the laboratory, with its potential to suppress extremes of behavior (e.g., Foster et al., 2008; cf. Heyman, 2001). Furthermore, the constraints of controlled laboratory observation limit the extent of sampling and observation of sequencing that can occur. These constraints make it difficult to discern the temporal direction of the association between marital interactions and alcohol involvement, which sometimes emerge over several days (Dunn et al., 1987). Daily process methods coupled with IVR technology can sample interactions sequentially over extended periods of time and have the potential to clarify the temporal direction of the reciprocal associations between marital behaviors and alcohol involvement.
Second, research showed that alcohol behavioral couples therapy reduced alcohol involvement among males and females with AUDs (McCrady et al., 2009; O'Farrell and Fals-Stewart, 2003). However, the mechanisms for the beneficial effects of couple-level interventions are not known. Collecting valid and reliable data on marital interactions from both partners is essential to the success of this work, yet self and collateral reports are by their nature distorted both by the time since an interaction took place and by their coloring of it over time. The utilization of daily IVR assessments would reduce these confounds and sources of error and could clarify the mechanisms underlying the beneficial effects of couple-level interventions.

\section{ACKNOWLEDGMENTS}

This research was supported by National Institute on Alcohol Abuse and Alcoholism (NIAAA) Grant R21 AA015105. We thank Janie Slayden, Jenna Johnson, Ann Mooney, Harriet Potts, Dr. Khamis Abu-Hasaballah, Dr. Kirk Brower, Dr. Stephen Strobbe, and the staff and clinicians of the University of Michigan Addiction Treatment Services (UMATS) for their assistance with this research.

\section{REFERENCES}

Abu-Hasaballah K, James A, Aseltine RH Jr (2007) Lessons and pitfalls of interactive voice response in medical research. Contemp Clin Trials 28:593602.

Agresti A (1996) An Introduction to Categorical Data Analysis. John Wiley \& Sons, Inc., New York.

Amato PR, Previti D (2003) People's reasons for divorcing: gender, social class, the life course, and adjustment. J Fam Issues 24:602-626.

Amato PR, Rogers SJ (1997) A longitudinal study of marital problems and subsequent divorce. J Marriage Fam 59:612-624.

Armeli S, Todd M, Mohr C (2005) A daily process approach to individual differences in stress-related alcohol use. J Pers 73:1-30.

Bolger N, Davis A, Rafaeli E (2003) Diary methods: capturing life as it is lived. Annu Rev Psychol 54:579-616.

Cherpitel CJ (2000) A brief screening instrument for problem drinking in the emergency room: the RAPS4. J Stud Alcohol 61:447-449.

Cherpitel CJ (2002) Screening for alcohol problems in the U.S. general population: comparison of the CAGE, RAPS4, and RAPS4-QF by gender, ethnicity, and service utilization. Alcohol Clin Exp Res 26:1686-1691.

Cohen J (1960) A coefficient of agreement for nominal scales. Educ Psychol Meas 20:37-46.

Collins RL, Kashdan TB, Gollnisch G (2003) The feasibility of using cellular phones to collect ecological momentary assessment data: application to alcohol consumption. Exp Clin Psychopharmacol 11:73-78.

Corkrey R, Parkinson L (2002) Interactive voice response: review of studies 1989-2000. Behav Res Methods 34:342-353.

Cranford JA, Shrout PE, Iida M, Rafaeli E, Yip T, Bolger N (2006) A procedure for evaluating sensitivity to within-person change: can mood measures in diary studies detect change reliably? Pers Soc Psychol Bull 32:917-929.

Dunn NJ, Jacob T, Hummon N, Seilhamer RA (1987) Marital instability in alcoholic-spouse relationships as a function of drinking pattern and location. J Abnorm Psychol 96:99-107.

Dunn NJ, Seilhamer RA, Jacob T, Whalen M (1992) Comparisons of retrospective and current reports of alcoholics and their spouses on drinking behavior. Addict Behav 17:543-555.

Fals-Stewart W, Leonard KE, Birchler GR (2005) The occurrence of male-to-female intimate partner violence on days of men's drinking: the 
moderating effects of antisocial personality disorder. J Consult Clin Psychol 73:239-248.

Floyd FJ, Cranford JA, Daugherty MK, Fitzgerald HE, Zucker RA (2006) Marital interaction in alcoholic and nonalcoholic couples: alcoholic subtype variations and wives' alcoholism status J Abnorm Psychol 115:121-130.

Foster DA, Caplan RD, Howe GW (1997) Representativeness of observed couple interaction: couples can tell, and it does make a difference. Psychol Assess 9:285-294.

Green AS, Rafaeli E, Bolger N, Shrout PE, Reis HT (2006) Paper or plastic? Data equivalence in paper and electronic diaries Psychol Methods 11:87105

Helzer JE, Badger GJ, Rose GL, Mongeon JA, Searles JS (2002) Decline in alcohol consumption during two years of daily reporting. J Stud Alcohol 63:551-558.

Helzer JE, Rose GL, Badger GJ, Searles JS, Thomas CS, Lindberg SA, Guth S (2008) Using interactive voice response to enhance brief alcohol intervention in primary care settings. J Stud Alcohol Drugs 69:251-258.

Heyman RE (2001) Observation of couple conflicts: clinical assessment applications, stubborn truths, and shaky foundations. Psychol Assess 13:5-35.

Jacob T, Leonard KE (1988) Alcoholic-spouse interaction as a function of alcoholism subtype and alcohol consumption interaction. J Abnorm Psychol 97:231-237.

Kenny DA, Kashy DA, Cook WL (2006) Dyadic Data Analysis. Guilford, New York, NY.

Kessler RC, Walters EE, Forthofer MS (1998) The social consequences of psychiatric disorders, III: probability of marital stability. Am J Psychiatry 155:1092-1096.

de Koning E, Weiss RL (2002) The Relational Humor Inventory: functions of humor in close relationships. Am J Fam Ther 30:1-18.

Kranzler HR, Abu-Hasaballah K, Tennen H, Feinn R, Young K (2004) Using daily Interactive Voice Response technology to measure drinking and related behaviors in a pharmacotherapy study. Alcohol Clin Exp Res 28:1060-1064.

Leigh BC (2000) Using daily reports to measure drinking and drinking patterns. J Subst Abuse 12:51-65.

Leonard KE, Eiden RD (2007) Marital and family processes in the context of alcohol use and alcohol disorders. Annu Rev Clin Psychol 3:285-310.

Leonard KE, Roberts LJ (1998) Marital aggression, quality, and stability in the first year of marriage: findings from the Buffalo Newlywed Study, in The Developmental Course of Marital Dysfunction (Bradbury TN ed), pp. 44-73. Cambridge University Press, New York, NY.

Leonard KE, Rothbard JC (1999) Alcohol and the marriage effect. J Stud Alcohol 13:139-146.

Litt MD, Cooney NL, Morse P (1998) Ecological momentary assessment (EMA) with treated alcoholics: methodological problems and potential solutions. Health Psychol 17:48-52.

Manne S, Ostroff J, Rini C, Fox K, Goldstein L, Grana G (2004) The interpersonal process model of intimacy: the role of self-disclosure, partner disclosure, and partner responsiveness in interactions between breast cancer patients and their partners. J Fam Psychol 18:589-599.

Marshal MP (2003) For better or for worse? The effects of alcohol use on marital functioning Clin Psychol Rev 23:959-997.

McCrady BS, Epstein EE, Cook S, Jensen N, Hildebrandt T (2009) A randomized trial of individual and couple behavioral alcohol treatment for women. J Consult Clin Psychol 77:243-256.

McCrady BS, Epstein EE, Kahler CW (2004) Alcoholics anonymous and relapse prevention as maintenance strategies after conjoint behavioral alcohol treatment for men: 18-month outcomes. J Consult Clin Psychol 72:870 878.

McCrady BS, Noel NE, Abrams DB, Stout RL (1986) Comparative effectiveness of three types of spouse involvement in outpatient behavioral alcoholism treatment. J Stud Alcohol 47:459-467.

McNair DM, Lorr M, Droppleman LF (1992) EdITS Manual for the Profile of Mood States. Educational and Industrial Testing Service, San Diego, CA.

Merrilees CE, Goeke-Morey M, Cummings EM (2008) Do event-contingent diaries about marital conflict change marital interactions? Behav Res Ther 46:253-262.
Miller WR, Tonigan JS, Longabaugh R (1995) The Drinker Inventory of Consequences (DrInC): an Instrument for Assessing Adverse Consequences of Alcohol Abuse. Project MATCH Monograph Series, Vol. 4. DHHS Publication No. 95-3911. NIAAA, Rockville, MD.

Mundt JC, Perrine MW, Searles JS, Walter D (1995) An application of interactive voice response (IVR) technology to longitudinal studies of daily behavior. Behav Res Methods 27:351-357.

Murphy CM, O'Farrell TJ (1996) Marital violence among alcoholics. Curr Dir Psychol Sci 5:183-186.

National Institute on Alcohol Abuse and Alcoholism, 2003, October 16. Task Force on Recommended Alcohol Questions - National Council on Alcohol Abuse and Alcoholism Recommended Sets of Alcohol Consumption Questions - October 15-16, 2003. Available at: http://www.niaaa.nih.gov/ Resources/ResearchResources/TaskForce.htm. Accessed December 15, 2005.

National Institute on Alcohol Abuse and Alcoholism (2004) NIAAA Council Approves Definition of Binge Drinking. NIAAA Newsletter, No. 3. National Institute on Alcohol Abuse and Alcoholism, Bethesda, MD.

O'Farrell TJ, Fals-Stewart W (2003) Alcohol abuse. J Marital Fam Ther 29:121-146.

Perrine MW, Mundt JC, Searles JS, Lester LS (1995) Validation of daily selfreported alcohol consumption using interactive voice response (IVR) technology. J Stud Alcohol 56:487-490.

Poikolainen K, Karkkainen P (1983) Diary gives more accurate information about alcohol consumption than questionnaire. Drug Alcohol Depend 11:209-216.

Prescott CA, Kendler KS (2001) Associations between marital status and alcohol consumption in a longitudinal study of female twins. J Stud Alcohol 62:589-604.

Rafaeli E, Cranford JA, Green AS, Shrout PE, Bolger N (2008) The good and bad of relationships: how social hindrance and social support affect relationship feelings in daily life. Pers Soc Psychol Bull 34:1703-1718.

Raudenbush SW, Bryk AS (2002) Hierarchical Linear Models: Applications and Data Analysis Methods, 2nd ed. Sage, Thousand Oaks, CA.

Rohsenow DJ (1982) Social anxiety, daily moods, and alcohol use over time among heavy social drinking men. Addict Behav 7:311-315.

Russell M, Martier SS, Sokol RJ, Mudar P (1994) Screening for pregnancy risk-drinking. Alcohol Clin Exp Res 18:1156-1161.

SAS Institute Inc. (2008) The GLIMMIX Procedure. SAS Institute Inc, Cary, NC.

Saunders JB, Aasland OG, Babor TF, de la Fuente JR (1993) Development of the Alcohol Use Disorders Identification Test (AUDIT): WHO collaborative project on early detection of persons with harmful alcohol consumption: II. Addiction 88:791-804.

Schuckit MA, Tipp JE, Bucholz KK, Nurnberger JI, Hesselbrock VM, Crowe RR, Kramwer J (1997) The life-time rates of three major mood disorders and four major anxiety disorders in alcoholics and controls. Addiction 92:1289-1304.

Schulz MS, Cowan PA, Pape Cowan C, Brennan RT (2004) Coming home upset: gender, marital satisfaction, and the daily spillover of workday experience into couple interactions. J Fam Psychol 18:250-263.

Searles JS, Helzer JE, Walter DE (2000) Comparison of drinking patterns measured by daily reports and timeline follow back. Psychol Addict Behav 14:277-286.

Searles JS, Perrine MW, Mundt JC, Helzer JE (1995) Self-report of drinking using touch-tone telephone: extending the limits of reliable daily contact. J Stud Alcohol 56:375-382.

Shiffman S, Stone AA, Hufford MR (2008) Ecological momentary assessment. Annu Rev Clin Psychol 4:1-32.

Simpson TL, Kivlahan DR, Bush KR, McFall ME (2005) Telephone selfmonitoring among alcohol use disorder patients in early recovery: a randomized study of feasibility and measurement reactivity. Drug Alcohol Depend 79:241-250.

Stone AA, Schwartz JE, Neale JM, Shiffman S, Marco CA, Hickcox M, Paty J, Porter LS, Cruise LJ (1998) A comparison of coping assessed by ecological momentary assessment and retrospective recall. J Pers Soc Psychol 74:1670-1680. 
Stone AA, Shiffman S (2002) Capturing momentary, self-report data: a proposal for reporting guidelines. Ann Behav Med 24:236-243.

Stone AA, Shiffman S, Schwartz JE, Broderick JE, Hufford MR (2002) Patient non-compliance with paper diaries. BMJ 324:1193-1194.

Stritzke WGK, Dandy J, Durkin K, Houghton S (2005) Use of interactive voice response (IVR) technology in health research with children. Behav Res Methods 37:119-126.

Tennen H, Affleck G (2002) The challenge of capturing daily processes at the interface of social and clinical psychology. J Soc Clin Psychol 21:610-627.

Tennen H, Affleck G, Armeli S (2003) Daily processes in health and illness, in Social Psychological Foundations of Health and Illness (Suls J, Wallston KA eds), pp. 495-529. Blackwell Publishing, Malden, MA.

Tennen H, Affleck G, Armeli S, Carney MA (2000) A daily process approach to coping: linking theory, research, and practice. Am Psychol 55:626-636.

Tennen H, Affleck G, Coyne JC, Larsen RJ, DeLongis A (2006) Paper and plastic in daily diary research: comment on Green, Rafaeli, Bolger, Shrout, and Reis (2006). Psychol Methods 11:112-118.

Tucker JA, Foushee HR, Black BC, Roth DL (2007) Agreement between prospective interactive voice response self-monitoring and structured retrospective reports of drinking and contextual variables during natural resolution attempts. J Stud Alcohol Drugs 68:538-542.

Uchalik DC (1979) A comparison of questionnaire and self-monitored reports of alcohol intake in a nonalcoholic population. Addict Behav 4:409-413.

Vinokur AD, Price RH, Caplan RD (1996) Hard times and hurtful partners: how financial strain affects depression and relationship satisfaction of unemployed persons and their spouses. J Pers Soc Psychol 71:166179.

Zucker RA (1994) The development of alcohol problems: exploring the biopsychosocial matrix of risk, in Pathways to Alcohol Problems and Alcoholism: A Developmental Account of the Evidence for Multiple Alcoholisms and for Contextual Contributions to Risk (Howard J, Boyd GM eds), pp 255289. U.S. Department of Health and Human Services, Rockville, MD.

Zucker RA (2008) Anticipating problem alcohol use developmentally from childhood into middle adulthood: What have we learned? Addiction 103:100-108.

Zucker RA, Donovan JE, Masten AS, Mattson ME, Moss HB (2008) Early developmental processes and the continuity of risk for underage drinking and problem drinking. Pediatrics 121:S252-S272.

Zucker RA, Fitzgerald HE, Moses HD (1995) Emergence of alcohol problems and the several alcoholisms: a developmental perspective on etiologic theory and life course trajectory, in Developmental Psychopathology: Risk, Disorder, and Adaptation Vol. 2 (Cicchetti D, Cohen DJ, eds), pp. 677-711. John Wiley \& Sons, New York.

Zucker RA, Fitzgerald HE, Refior SK, Puttler LI, Pallas DM, Ellis DA (2000) Children of addiction: research, health, and public policy issues, in The Clinical and Social Ecology of Childhood for Children of Alcoholics: Description of a Study and Implications for a Differentiated Social Policy (Fitzgerald HE, Lester BM, Zuckerman BS eds), pp. 109-141. RoutledgeFalmer Press, New York. 